\title{
A RIEMANNIAN INVARIANT AND ITS APPLICATIONS TO EINSTEIN MANIFOLDS
}

\author{
BANG-YEN CHEN
}

\begin{abstract}
We introduce a Riemannian invariant and establish general optimal inequalities involving the invariants and the squared mean curvature for Einstein manifolds isometrically immersed in real space forms. We show that these inequalities do not hold for arbitrary submanifolds in real space forms in general. We also provide some immediate applications of the inequalities.
\end{abstract}

\section{INTRODUCTION.}

According to the celebrated embedding theorem of Nash [9], every Riemannian manifold can be isometrically embedded in some Euclidean space with sufficiently high codimension. An immediate question concerning Nash's embedding theorem is the following. PROBLEM 1. What can we do with Nash's embedding theorem? In other words, what can we do with arbitrary Euclidean submanifolds of arbitrary high codimension if "no local or global assumption" were imposed on the submanifold?

The Nash theorem was established in the hope that if Riemannian manifolds could always be regarded as Riemannian submanifolds, this would then yield the opportunity to use extrinsic help. As observed by Gromov [7], this hope has not materialised however. The main reason for this is due to the lack of control on the extrinsic properties of the submanifolds by the known intrinsic data (see [10]).

In order to overcome such difficulty, one needs to introduce new types of Riemannian invariants different from the known ones such as Ricci curvature and scalar curvature. One also needs to establish general optimal relationships between the main extrinsic invariants with the new type of intrinsic invariants on the submanifolds.

The author was able to provide general optimal solutions to Problem 1 in terms of the $\delta$-invariants $\delta\left(n_{1}, \ldots, n_{k}\right)$ he introduced in [2]. In the context of Nash's theorem, he has established for all these invariants optimal general inequalities involving the main extrinsic invariant; namely, the squared mean curvature. These general inequalities provide prima controls on the most important extrinsic curvature by the initial intrinsic data of the Riemannian manifolds. As results, he was able to discover new intrinsic spectral Received 22nd October, 2003

Copyright Clearance Centre, Inc. Serial-fee code: 0004-9727/04 \$A2.00+0.00. 
properties of homogeneous spaces via Nash's theorem. Such results extend a well-known theorem of Nagano [8].

Following the same line of thought, the author asked in [3] the following basic question.

Problem 2. Let $\mathfrak{F}$ be a family of Riemannian manifolds associated with a given special structure. What are the special relationships between the intrinsic invariants on manifolds $M$ in $\mathfrak{F}$ and the main extrinsic invariants of $M$ if $M$ were immersed isometrically in an arbitrary Euclidean space (or, more generally, in an arbitrary space form) and if no local or global assumption were imposed on the submanifolds?

In $[3,4,5,6]$, the author was able to establish optimal solutions to Problem 2 for the family of warped product manifolds with warped product structure and for the family of conformally flat manifolds with conformally flat structure.

The purpose of this paper is to provide further optimal solutions to Problem 2 for another important family of Riemannian manifolds; namely, the family of Einstein manifolds. In order to do so, we introduce some new Riemannian invariants and establish sharp general relationships between the invariants and the squared mean curvature for Einstein submanifolds. We show that these inequalities do not hold for non-Einstein submanifolds in general. Several immediate applications are also provided.

\section{Preliminaries.}

Let $M$ be a Riemannian $n$-manifold. Denote by $K(\pi)$ the sectional curvature of $M$ associated with a plane section $\pi \subset T_{p} M, p \in M$. For each unit tangent vector $X$ of $M$ at $p$, the $\operatorname{Ricci}$ curvature $\operatorname{Ric}(X, X)$ is defined by

$$
\operatorname{Ric}(X, X)=\sum_{j=2}^{n} K\left(X \wedge e_{j}\right),
$$

where $\left\{e_{1}, \ldots, e_{n}\right\}$ is an orthonormal basis of $T_{p} M$ with $e_{1}=X$.

If $M$ is an Einstein manifold, $\operatorname{Ric}(X, X)$ is independent of the choice of the unit vector $X$. In this case, we denote the common value simply by Ric.

The scalar curvature $\tau$ of $M$ is given by

$$
\tau(p)=\sum_{i<j} K\left(e_{i} \wedge e_{j}\right)
$$

Now, suppose that $M$ is isometrically immersed in a Riemannian $m$-manifold $R^{m}(c)$ of constant curvature $c$. Let $h$ and $A$ denote the second fundamental form and the shape operator of the submanifold.

Let $\left\{h_{i j}^{r}\right\}, 1 \leqslant i, j \leqslant n ; n+1 \leqslant r \leqslant m$, denote the coefficients of the second fundamental form with respect to an orthonormal frame field $e_{1}, \ldots, e_{n}, e_{n+1}, \ldots, e_{m}$ so that $e_{1}, \ldots, e_{n}$ are tangent to $M$ and thus $e_{n+1}, \ldots, e_{m}$ are normal to $M$. 
The mean curvature vector is given by

$$
\vec{H}=\frac{1}{n} \text { trace } h
$$

and squared mean curvature $H^{2}$ is $\langle\vec{H}, \vec{H}\rangle$, where $\langle$,$\rangle is the inner product.$

The equation of Gauss of $M$ in $R^{m}(c)$ is

$$
\begin{aligned}
R(X, Y ; Z, W)=c\{\langle X, W\rangle\langle Y, Z\rangle-\langle X, Z\rangle\langle Y, W\rangle\} & \\
& +\langle h(X, W), h(Y, Z)\rangle-\langle h(X, Z), h(Y, W)\rangle .
\end{aligned}
$$

A submanifold $M$ is called pseudo-umbilical if $\vec{H}$ is nonzero and the shape operator $A_{\vec{H}}$ at $\vec{H}$ is proportional to the identity map.

Let $n$ be a natural number greater than or equal to 2 . If $k$ natural numbers $n_{1}, \ldots, n_{k}$ satisfy $n_{1}+\cdots+n_{k}=n$, then $\left(n_{1}, \ldots, n_{k}\right)$ is called a partition of $n$.

We need the following general algebraic lemma from [5] for later use.

LEMMA 1. Let $a_{1}, \ldots, a_{n}$ be $n$ real numbers and let $k$ be an integer in $[2, n-1]$. Then, for any partition $\left(n_{1}, \ldots, n_{k}\right)$ of $n$, we have

$\sum_{1 \leqslant i_{1}<j_{1} \leqslant n_{1}} a_{i_{1}} a_{j_{1}}+\cdots+\sum_{n_{1} \cdots+n_{k-1}+1 \leqslant i_{1}<j_{1} \leqslant n} a_{i_{k}} a_{j_{k}} \geqslant \frac{1}{2 k}\left\{\left(a_{1}+\cdots+a_{n}\right)^{2}-k\left(a_{1}^{2}+\cdots+a_{n}^{2}\right)\right\}$, with the equality holding if and only if we have

$$
a_{1}+\cdots+a_{n_{1}}=\cdots=a_{n_{1}+\cdots+n_{k-1}+1}+\cdots+a_{n} .
$$

\section{RiEMANNIAN INVARIANTS.}

Let $p$ be a point in a Riemannian $n$-manifold $M$ and $q$ a natural number $\leqslant n / 2$. For a given point $p \in M$, let $\pi_{1}, \ldots, \pi_{q}$ be $q$ mutually orthogonal plane sections in $T_{p} M$. Define the invariant $K_{q}^{\text {inf }}(p)$ to be the infimum of the average of the sectional curvatures $K\left(\pi_{1}\right), \ldots, K\left(\pi_{q}\right)$, that is,

$$
K_{q}^{\mathrm{inf}}(p)=\inf _{\pi_{1} \perp \cdots \perp \pi_{q}} \frac{K\left(\pi_{1}\right)+\cdots+K\left(\pi_{q}\right)}{q}
$$

where $\pi_{1}, \ldots, \pi_{q}$ run over all mutually orthogonal $q$ plane sections in $T_{p} M$.

For each natural number $q \leqslant n / 2$, we define the Riemannian invariant $\delta_{q}^{\text {Ric }}$ on $M$ by

$$
\delta_{q}^{\mathrm{Ric}}(p)=\sup _{X \in T_{p}^{1} M} \operatorname{Ric}(X, X)-\frac{2 q}{n} K_{q}^{\mathrm{inf}}(p),
$$

where $n=\operatorname{dim} M, X$ runs over all unit vectors in $T_{p}^{1} M:=\left\{X \in T_{p} M:|X|=1\right\}$.

REMARK 1. Although the invariants $\delta_{q}^{\text {Ric }}, q \leqslant n / 2$, are similar to the $\delta$-invariant $\delta(2)$, the invariants $\delta_{q}^{\text {Ric }}$ and $\delta(2)$ are different in nature.

For example, for Einstein 4-manifolds, we have

$$
\delta_{2}^{\text {Ric }}=\operatorname{Ric}-K_{2}^{\text {inf }}(\pi), \quad \delta(2)=\tau-\inf K(\pi) .
$$

where $\pi$ runs over all plane sections at each given point. 


\section{The first main Result.}

The following theorem provides an optimal general solution to Problem 2 for Einstein manifolds.

THEOREM 1. For any integer $k \geqslant 2$ and any isometric immersion of an Einstein $2 k$-manifold $M$ into a Riemannian $m$-manifold $R^{m}(c)$ of constant curvature $c$ with arbitrary codimension, we have

$$
\delta_{k}^{\text {Ric }} \leqslant 2(k-1)\left(H^{2}+c\right)
$$

The equality sign of (4.1) holds identically if and only if one of the following two cases occurs:

(1) $M$ is a minimal Einstein submanifold such that, with respect to some suitable orthonormal frame $\left\{e_{1}, \ldots, e_{2 k}, e_{2 k+1}, \ldots, e_{m}\right\}$, the shape operator of $M$ takes the following form:

$$
A_{r}=\left(\begin{array}{ccc}
A_{1}^{r} & & 0 \\
& \ddots & \\
0 & & A_{k}^{r}
\end{array}\right), r=2 k+1, \ldots, m,
$$

where $A_{j}^{r}, j=1, \ldots, k$, are symmetric $2 \times 2$ submatrices satisfying $\operatorname{trace}\left(A_{1}^{r}\right)=\cdots$ $=\operatorname{trace}\left(A_{k}^{r}\right)=0$.

(2) $M$ is a pseudo-umbilical Einstein submanifold such that, with respect to some suitable orthonormal frame $\left\{e_{1}, \ldots, e_{2 k}, e_{2 k+1}, \ldots, e_{m}\right\}$, the shape operators takes the following form:

$$
A_{r}=\left(\begin{array}{ccc}
A_{1}^{r} & & 0 \\
& \ddots & \\
0 & & A_{k}^{r}
\end{array}\right), r=2 k+2, \ldots, m,
$$

where $A_{j}^{r}, j=1, \ldots, k$, are symmetric $2 \times 2$ submatrices satisfying trace $\left(A_{1}^{r}\right)=\ldots$ $=\operatorname{trace}\left(A_{k}^{r}\right)=0$.

PRoOF: Let $\phi: M \rightarrow R^{m}(c)$ be an isometric immersion of an Einstein manifold $M$ into a real space form $R^{m}(c)$. Suppose that $\pi_{1}, \ldots, \pi_{k}$ are $k$ mutually orthogonal plane sections at $p$. We choose an orthonormal basis $e_{1}, \ldots, e_{2 k}$ of $T_{p} M$ such that

$$
\pi_{1}=\operatorname{Span}\left\{e_{1}, e_{2}\right\}, \ldots, \pi_{k}=\operatorname{Span}\left\{e_{2 k-1}, e_{2 k}\right\}
$$

We choose an orthonormal basis $e_{2 k+1}, \ldots, e_{m}$ of the normal space $T_{p}^{\perp} M$ such that $e_{2 k+1}$ is parallel to $\vec{H}$ at $p$ whenever $\vec{H} \neq 0$ at $p$. If $\vec{H}=0$ at $p$, we have no restriction on $e_{2 k+1}$.

From the equation of Gauss, we have

$$
2 \tau=4 k^{2} H^{2}-\|h\|^{2}+2 k(2 k-1) c
$$


where $\|h\|^{2}$ denotes the squared norm of $h$.

If we put

$$
\varphi=2 \tau-2 k(2 k-1) c-4 k(k-1) H^{2},
$$

then (4.2) is nothing but

$$
4 k H^{2}=\varphi+\|h\|^{2} .
$$

We may express (4.4) as

$$
\left(\sum_{i=1}^{2 k} a_{i}\right)^{2}-k \sum_{i=1}^{2 k}\left(a_{i}\right)^{2}=k \varphi+k \sum_{i \neq j}\left(h_{i j}^{2 k+1}\right)^{2}+k \sum_{r=2 k+2}^{m} \sum_{i, j=1}^{2 k}\left(h_{i j}^{r}\right)^{2} .
$$

where $a_{j}=h_{j j}^{2 k+1}, j=1, \ldots, 2 k$. Hence, we may applying Lemma 1 to (4.5) to obtain that

$$
a_{1} a_{2}+a_{3} a_{4}+\cdots+a_{2 k-1} a_{2 k} \geqslant \frac{\varphi}{2}+\sum_{1 \leqslant i<j \leqslant 2 k}\left(h_{i j}^{2 k+1}\right)^{2}+\frac{1}{2} \sum_{r=2 k+2}^{m} \sum_{i, j=1}^{2 k}\left(h_{i j}^{r}\right)^{2},
$$

with the equality sign holding if and only if we have

$$
a_{1}+a_{2}=\cdots=a_{2 k-1}+a_{2 k} .
$$

On the other hand, the equation of Gauss yields

$$
K\left(\pi_{j}\right)=c+\sum_{r=2 k+1}^{m}\left\{h_{2 j-12 j-1}^{r} h_{2 j 2 j}^{r}-\left(h_{2 j-12 j}^{r}\right)^{2}\right\}
$$

Thus, by combining this equation with inequality (4.6), we discover that

$$
\begin{aligned}
\tau-K\left(\pi_{1}\right) & -\cdots-K\left(\pi_{k}\right) \\
\leqslant & -\frac{\varphi}{2}-k c-\sum_{r=2 k+1}^{m} \sum_{j \neq 1,2}\left\{\left(h_{1 j}^{r}\right)^{2}+\left(h_{2 j}^{r}\right)^{2}\right\}-\cdots \\
& -\sum_{r=2 k+1}^{m} \sum_{j \neq 2 k-1,2 k}\left\{\left(h_{2 k-1 j}^{r}\right)^{2}+\left(h_{2 k j}^{r}\right)^{2}\right\} \\
& -\frac{1}{2} \sum_{r=n+2}^{m}\left\{\left(h_{11}^{r}+h_{22}^{r}\right)^{2}+\cdots+\left(h_{2 j-12 j-1}^{r}+h_{2 k 2 k}^{r}\right)^{2}\right\} \\
\leqslant & -\frac{\varphi}{2}-k c .
\end{aligned}
$$

Therefore, by combining (4.3) and (4.8), we obtain

$$
\tau-K\left(\pi_{1}\right)-\cdots-K\left(\pi_{k}\right) \leqslant 2 k(k-1)\left(H^{2}+c\right) .
$$


Since $M$ is an Einstein $2 k$-manifold, we have $\tau=k$ Ric. Hence, by applying (3.1) and (4.9), we discover that

$$
\text { Ric }-K_{k}^{\text {inf }} \leqslant 2(k-1)\left(H^{2}+c\right) .
$$

Thus, we obtain (4.1) by combining (3.2) and (4.10).

If the equality sign of (4.1) holds identically, then all of the inequalities in (4.6) and (4.8) are actually equalities. From those we conclude that there exist a suitable orthonormal frame $\left\{e_{1}, \ldots, e_{2 k}\right\}$ of the tangent bundle and an orthonormal frame $\left\{e_{2 k+1}, \ldots, e_{m}\right\}$ of the normal bundle such that the shape operator with respect these frames takes the form:

$$
A_{r}=\left(\begin{array}{ccc}
A_{1}^{r} & & 0 \\
& \ddots & \\
0 & & A_{k}^{r}
\end{array}\right), r=2 k+1, \ldots, m,
$$

where $A_{j}^{r}, j=1, \ldots, k$, are symmetric $2 \times 2$ submatrices satisfying

$$
\operatorname{trace}\left(A_{1}^{r}\right)=\cdots=\operatorname{trace}\left(A_{k}^{r}\right)
$$

for $r=2 k+1, \ldots, m$.

If $M$ is a minimal submanifold of $R^{m}(c)$, we obtain from $(4.12)$ that trace $\left(A_{j}^{r}\right)=0$ for $r=2 k+1, \ldots, m$ and $j=1, \ldots, k$. This gives Case (1) of the theorem.

If $M$ is non-minimal, we choose $e_{2 k+1}$ parallel to $\vec{H}$. So, we find from (4.12) that

$$
\operatorname{trace}\left(A_{1}^{r}\right)=\cdots=\operatorname{trace}\left(A_{k}^{r}\right)=0
$$

for $r=2 k+2, \ldots, m$. Moreover, because $A_{2 k+1}$ takes the form of (4.11), we may further choose $e_{1}, \ldots, e_{2 k}$ diagonalising $A_{2 k+1}$. Hence, we have

$$
A_{j}^{2 k+1}=\left(\begin{array}{cc}
a_{2 j-1} & 0 \\
0 & a_{2 j}
\end{array}\right), j=1, \ldots, k,
$$

where $a_{1}, \ldots, a_{2 k}$ satisfy

$$
a_{1}+a_{2}=\cdots=a_{2 k-1}+a_{2 k}=2 a \text {. }
$$

for some function $a$.

So, by applying (4.11)-(4.15), we find

$$
\begin{aligned}
\operatorname{Ric}\left(e_{2 j-1}, e_{2 j-1}\right) & =K\left(\pi_{j}\right)+2(k-1) a a_{2 j-1}+2(k-1) c \\
\operatorname{Ric}\left(e_{2 j}, e_{2 j}\right) & =K\left(\pi_{j}\right)+2(k-1) a a_{2 j}+2(k-1) c .
\end{aligned}
$$

Since $M$ is Einsteinian, (4.16) implies that

$$
\begin{gathered}
a_{1}=a_{2}=\cdots=a_{2 k-1}=a_{2 k}=a, \\
K\left(\pi_{1}\right)=\cdots=K\left(\pi_{k}\right)=K_{k}^{\mathrm{inf}} .
\end{gathered}
$$


From (4.17) we know that $M$ is a pseudo-umbilical Einstein submanifold of $R^{m}(c)$.

The converse is easy to verify.

REMARK 2. Inequality (4.1) is sharp. For examples, each hypersphere $S^{2 k}$ of $\mathbb{E}^{2 k+1}$ and the standard embedding of the Riemannian product of two unit spheres $S^{2}(1) \times S^{2}(1)$ in $\mathbb{E}^{6}$ are umbilical and pseudo-umbilical Einstein submanifolds of Euclidean spaces which satisfy the equality case of (4.1).

REMARK 3. The minimal Clifford torus:

$$
S^{2}(1) \times S^{2}(1) \subset S^{5}(1)
$$

is a minimal submanifold of $S^{5}(1)$ which satisfy the equality case of (4.1) with $c=1$.

This example and the examples given in Remark 2 show that both Case (1) and Case (2) of Theorem 1 do occur.

REMARK 4. The following example shows that inequality (4.1) does not hold for arbitrary submanifolds in general.

EXAMPLE 1. Consider the spherical hypercylinder:

$$
M:=S^{2}(1) \times \mathbb{E}^{2 k-2} \subset \mathbb{E}^{2 k+1} .
$$

We have $\delta_{k}^{\text {Ric }}=1$ and $H^{2}=1 / k^{2}$ on $M$ which imply that

$$
\delta_{k}^{\mathrm{Ric}}=1>\frac{2(k-1)}{k^{2}}=2(k-1) H^{2}
$$

for $k \geqslant 2$.

\section{THE SECOND MAIN RESUlT.}

THEOREM 2. Let $\phi: M \rightarrow R^{m}(c)$ be an isometric immersion of an Einstein nmanifold $M$ into a Riemannian $m$-manifold $R^{m}(c)$ of constant curvature $c$ with arbitrary codimension. Then, for every natural number $q<n / 2$, we have

$$
\delta_{q}^{\text {Ric }} \leqslant \frac{n(n-q-1)}{n-q} H^{2}+\left(n-1-\frac{2 q}{n}\right) c .
$$

The equality sign of (5.1) holds identically if and only if $M$ is a totally geodesic submanifold.

Proof: Let $\phi$ be an isometric immersion of an Einstein $n$-manifold $M$ into $R^{m}(c)$. Assume that $q$ is a natural number $<n / 2$ and $p$ is a point in $M$. Consider $q$ mutually orthogonal plane sections $\pi_{1}, \ldots, \pi_{q}$ of $M$ at $p$. We choose an orthonormal basis $\left\{e_{1}, \ldots, e_{n}\right\}$ of $T_{p} M$ such that $\pi_{j}$ is spanned by $e_{2 j-1}$ and $e_{2 j}$ for $j=1, \ldots, q$. 
From the equation of Gauss we find

$$
2 \tau=n^{2} H^{2}-\|h\|^{2}+n(n-1) c .
$$

If we put

$$
\eta=2 \tau-n(n-1) c-\frac{n^{2}(n-q-1)}{n-q} H^{2}
$$

then (5.2) becomes

$$
n^{2} H^{2}=(n-q)\left(\eta+\|h\|^{2}\right) .
$$

Equation (5.4) can be rewritten as

$$
\frac{1}{n-q}\left(\sum_{i=1}^{n} a_{i}\right)^{2}-\sum_{i=1}^{n}\left(a_{i}\right)^{2}=\eta+\sum_{i \neq j}\left(h_{i j}^{n+1}\right)^{2}+\sum_{r=n+2}^{m} \sum_{i, j=1}^{n}\left(h_{i j}^{r}\right)^{2}
$$

where $a_{j}=h_{j j}^{n+1}, j=1, \ldots, n$.

Since $(\overbrace{2, \ldots, 2}^{q \text { times }}, \overbrace{1, \ldots, 1}^{n-2 q \text { times }})$ is a partition of $n$, we may applying Lemma 1 to $(5.5)$ to obtain that

$$
a_{1} a_{2}+a_{3} a_{4}+\cdots+a_{2 q-1} a_{2 q} \geqslant \frac{\eta}{2}+\sum_{1 \leqslant i<j \leqslant 2 k}\left(h_{i j}^{2 k+1}\right)^{2}+\frac{1}{2} \sum_{r=2 k+2}^{m} \sum_{i, j=1}^{2 k}\left(h_{i j}^{r}\right)^{2},
$$

with the equality sign holding if and only if we have

$$
a_{1}+a_{2}=\cdots=a_{2 q-1}+a_{2 q}=a_{2 q+1}=\cdots=a_{n}
$$

Thus, by using inequality (5.6) and the equation of Gauss, we may find just like in the proof of Theorem 1 that

$$
\delta_{q}^{\mathrm{Ric}} \leqslant \frac{2}{n}\left(\tau-\frac{\eta}{2}-q c\right)
$$

Therefore, by combining (5.3) and (5.7), we obtain inequality (5.1).

If the equality sign of (5.1) holds identically, then by applying an argument similar to that given in the proof of Theorem 1 we know that the shape operator of $M$, with respect to some suitable orthonormal frame $\left\{e_{1}, \ldots, e_{n}, e_{n+1}, \ldots, e_{m}\right\}$, takes the form:

$$
A_{r}=\left(\begin{array}{cccc}
A_{1}^{r} & & & 0 \\
& \ddots & & \\
& & A_{k}^{r} & \\
0 & & & \mu_{r} I
\end{array}\right),
$$


for $r=n+1, \ldots, m$, where $I$ is the $(n-2 q) \times(n-2 q)$ identity matrix and $A_{j}^{r}, j=1, \ldots, k$, are symmetric $2 \times 2$ submatrices satisfying

$$
\operatorname{trace}\left(A_{1}^{r}\right)=\cdots=\operatorname{trace}\left(A_{k}^{r}\right)=\mu_{r} .
$$

CASE (i): $M$ is minimal. In this case, we have $\mu_{n+1}=\cdots=\mu_{m}=0$. Hence, (5.8) implies that $\operatorname{Ric}=\operatorname{Ric}\left(e_{n}, e_{n}\right)=(n-1) c$, since $M$ is Einsteinian.

On the other hand, it is well-known that the only $n$-dimensional minimal Einstein submanifolds in $R^{m}(c)$ with Ric $=(n-1) c$ are the totally geodesic ones (see [1]). Thus, $M$ is a totally geodesic submanifold.

CASE (ii): $M$ is non-minimal. In this case, we may choose $e_{n+1}$ parallel to $\vec{H}$. From (5.8) and (5.9) we find

$$
\operatorname{trace}\left(A_{1}^{r}\right)=\cdots=\operatorname{trace}\left(A_{k}^{r}\right)=0
$$

for $r=n+2, \ldots, m$. Moreover, because $A_{n+1}$ takes the form of (5.8), we may also choose $e_{1}, \ldots, e_{n}$ which diagonalise $A_{n+1}$. So, we have

$$
A_{j}^{n+1}=\left(\begin{array}{cc}
a_{2 j-1} & 0 \\
0 & a_{2 j}
\end{array}\right), j=1, \ldots, q,
$$

where $a_{1}, \ldots, a_{2 q}$ satisfy

$$
a_{1}+a_{2}=\cdots=a_{2 q-1}+a_{2 q}=\mu_{n+1} .
$$

By computing Ric $\left(e_{2 j-1}, e_{2 j-1}\right)$ and $\operatorname{Ric}\left(e_{2 j}, e_{2 j}\right)$ for $j=1, \ldots, q$ and by applying (5.8), (5.10), (5.11) and (5.12), we find

$$
\begin{aligned}
a_{1}=a_{2} & =\cdots=a_{2 q}=a, \quad a=\frac{\mu_{n+1}}{2}, \\
\operatorname{Ric}\left(e_{2 j}, e_{2 j}\right) & =K\left(\pi_{j}\right)+2(n-q-1) a^{2}+(n-2) c .
\end{aligned}
$$

By applying (5.8) and (5.10)-(5.13), we have

$$
\operatorname{Ric}\left(e_{n}, e_{n}\right)=4(n-q-1) a^{2}+(n-1) c .
$$

By comparing (5.14) and (5.15), we obtain

$$
K\left(\pi_{j}\right)=2(n-q-1) a^{2}+c .
$$

On the other hand, from (5.8), (5.10), (5.11) and (5.13), we also have

$$
K\left(\pi_{j}\right)=a^{2}+c-\frac{1}{2} \sum_{r=n+2}^{m}\left\|A_{j}^{r}\right\|^{2},
$$

where $\left\|A_{j}^{r}\right\|^{2}$ is the squared norm of $A_{j}^{r}$. Thus, by comparing (5.16) and (5.17), we conclude that $M$ is totally geodesic in $R^{m}(c)$ which is a contradiction. Consequently, the equality sign of (5.1) implies that $M$ is a totally geodesic submanifold of $R^{m}(c)$.

The converse is trivial. 
REMARK 5. The next example shows that inequality (5.1) does not hold for arbitrary submanifolds in general as well.

EXAMPLE 2. For the spherical hypercylinder: $S^{n-q}(1) \times \mathbb{E}^{q} \subset \mathbb{E}^{n+1}$, we have

$$
\delta_{q}^{\mathrm{Ric}}=n-q-1, H^{2}=\frac{(n-q)^{2}}{n^{2}}
$$

for $q<n / 2$ which imply that

$$
\delta_{q}^{\mathrm{Ric}}>\frac{n(n-q-1)}{n-q} H^{2} .
$$

\section{IMMEDIATE APPLICATIONS.}

From Theorems 1 and 2 we obtain immediately the following.

COROLlaRY 1. If a Riemannian manifold $M$ admits an isometric immersion into a Euclidean space which satisfies

$$
\delta_{q}^{\mathrm{Ric}}>\frac{n(n-q-1)}{n-q} H^{2}, \quad n=\operatorname{dim} M,
$$

for some natural number $q \leqslant n / 2$ at some point, then $M$ is not an Einstein manifold.

This corollary applies to a large family of Riemannian manifolds. The simplest cases are the following: $S^{2} \times \mathbb{E}^{2 k-2}$ and $S^{n-q} \times \mathbb{E}^{q}, q<n / 2$, cannot be Einsteinian. This follows immediately from Examples 1 and 2 and Corollary 1.

Theorems 1 and 2 also imply the following.

Corollary 2. If an Einstein $n$-manifold satisfies

$$
\delta_{q}^{\text {Ric }}>\left(n-1-\frac{2 q}{n}\right) c
$$

for some natural number $q \leqslant n / 2$ at some point, then it admits no minimal isometric immersion into $R^{m}(c)$ regardless of codimension.

Applying Theorem 1 and 2 we also have the following.

COROLlary 3 . Let $M$ be a compact Einstein $n$-manifold with finite fundamental group $\pi_{1}$ or with null first betti number, that is, $b_{1}=0$. If there is a natural number $q \leqslant n / 2$ such that $\delta_{q}^{\text {Ric }}>0$, then $M$ admits no Lagrangian isometric immersion into any complex n-torus or complex Euclidean $n$-space.

By applying Theorems 1 and 2, this corollary can be proved in a way similar to the proof of [2, Theorem 4.2 ]. 


\section{REFERENCES}

[1] B.Y. Chen, Geometry of submanifolds (M. Dekker, New York, 1973).

[2] B.Y. Chen, 'Some new obstructions to minimal and Lagrangian isometric immersions', Japan. J. Math. 26 (2000), 105-127.

[3] B.Y. Chen, 'On isometric minimal immersions from warped products into real space forms', Proc. Edinburgh Math. Soc. 45 (2002), 579-587.

[4] B.Y. Chen, 'Non-immersion theorems for warped products in complex hyperbolic spaces', Proc. Japan Acad. Ser. A Math. Sci. 79 (2002), 96-100.

[5] B.Y. Chen, 'A general optimal inequality for warped products in complex projective spaces and its applications', Proc. Japan Acad. Ser. A Math. Sci. 79 (2003), 89-94.

[6] B.Y. Chen, 'A general inequality for conformally flat submanifolds and its applications' (to appear).

[7] M. Gromov, 'Isometric immersions of Riemannian manifolds, Elie Cartan et les Mathématiques d'Aujourd'hui', Astérisque (1985), 129-133.

[8] T. Nagano, 'On the minimum eigenvalues of the Laplacians in Riemannian manifolds', Sci. Papers Coll. Gen. Edu. Univ. Tokyo 11 (1961), 177-182.

[9] J.F. Nash, 'The imbedding problem for Riemannian manifolds', Ann. of Math. 63 (1956), $20-63$.

[10] S.T. Yau, 'Mathematical research today and tomorrow', in Viewpoints of Seven Fields Medalists (Berlin, Heidelberg, New York, 1991), pp. 30-39.

Department of Mathematics

Michigan State University

East Lansing, MI 48824-1027

United States of America

e-mail: bychen@math.msu.edu 